\title{
Brown Heroin-Associated Candida albicans Ventriculitis and Endophthalmitis Treated with Voriconazole
}

\author{
Nora Elfiky Kelly Baldwin \\ Geisinger Medical Center, Department of Neurology, Danville, Pa., USA
}

\section{Keywords}

Chronic meningitis · Fungal meningitis · Ventriculitis · Candida albicans · Endophthalmitis .

Brown heroin

\begin{abstract}
Chronic meningitis and ventriculitis are defined as inflammatory pleocytoses in the cerebrospinal fluid (CSF) and ependyma that persists for at least 1 month without spontaneous resolution. Because the CSF communicates directly with the posterior compartments of the eye, fungal infections in the brain often cause secondary ophthalmologic complications. We report a 23-year-old male who presented to the emergency room with progressive severe headaches associated with insidious monocular vision loss. After extensive workup and a multidisciplinary team effort, the patient was diagnosed with ventriculitis and endogenous endophthalmitis. The etiology is suspected to be due to brown heroin use with secondary disseminated Candida albicans.

(C) 2016 The Author(s)

Published by S. Karger AG, Basel
\end{abstract}

\section{Introduction}

Chronic meningitis, ventriculitis, and mycotic endogenous endophthalmitis are difficult diagnoses to solidify due to the broad nature of the differential diagnosis and barriers to confirmatory testing. However, early diagnosis and accurate management are critical in re- 
ducing morbidity and mortality in patients. We describe a case of Candida albicans ventriculitis and endophthalmitis secondary to brown heroin abuse successfully treated with oral voriconazole.

Brown heroin is a base that necessitates the addition of an acid in order to create a salt rendering the heroin more suitable for injection. Typically, readily available forms of acid, such as lemon juice, citric acid, and vinegar are used. The addition of lemon juice has been implicated in multiple cases of disseminated candidiasis among brown heroin users [1].

\section{Case Presentation}

A 23-year-old Caucasian male presented to the emergency department with a 2-month history of daily headaches and a 3-week history of progressive vision loss in his left eye. The headaches were initially described as intermittent, holocephalic, and throbbing with associated photophobia. Over the course of several months, they increased in severity and became constant with an overlying sharp left-sided facial pain. Coinciding with a worsening headache, the patient also noted escalating visual changes in his left eye. On review of systems the patient also endorsed myalgias, generalized fatigue, night sweats, and nuchal rigidity, all of which were present for 1 month.

Social and family history was obtained, which revealed a previous incarceration and tobacco abuse. Although on initial questioning the patient denied illicit drug abuse, later in the hospitalization on repeat questioning the patient admitted to recent and remote intravenous brown heroin use. Additionally, the patient continued to deny any other illicit drug use.

Physical examination revealed a dilated, nonreactive left pupil with blurred disc margins and a visual acuity of 20/100 in the left eye. The patient was found to have diminished pinprick sensation in the trigeminal V2 and V3 distribution on the left side of his face. No other abnormalities were noted on physical examination.

Ophthalmology was consulted and a dilated eye exam revealed vitreous opacities with associated vitritis in a 'string of pearls' appearance, vitreous hemorrhage, cotton wool spots, and optic disc edema in his left eye (fig. 1). These findings were most concerning for Candida albicans endophthalmitis. Because of meningeal symptoms and subjective complaints of trigeminal neuralgia, magnetic resonance imaging (MRI) of the brain with and without contrast was completed. It revealed enhancement of the margins of the lateral, third, and fourth ventricle and dural enhancement around the left Meckel's cave (fig. 2). Subsequently, a lumbar puncture was performed revealing 7,775 WBC/cu mm (53\% neutrophils, $40 \%$ lymphocytes), 1,150 RBC/cu mm, $295 \mathrm{mg} / \mathrm{dl}$ protein, and $9 \mathrm{mg} / \mathrm{dl}$ glucose. Cerebrospinal fluid (CSF) studies were negative for bacterial meningitis antigen panel, cryptococcal antigen, herpes simplex virus PCR, and varicella zoster virus PCR. Gram stain and fungal cultures of the CSF were also unremarkable. Vitreal fluid samples revealed negative acid-fast bacilli. Serum studies were negative for Bartonella henselae antibodies, Haemophilus influenzae PCR, and syphilis studies including FTAbs.

Given the patient's clinical examination, laboratory testing, ophthalmologic findings, and history of brown heroin abuse, a diagnosis of Candida albicans ventriculitis, meningitis, and endogenous endophthalmitis was made. Systemic therapy with i.v. amphotericin B and fluconazole were initiated. Adverse effects of amphotericin B including severe shivering and tachycardia led to a pharmacologic change to oral voriconazole $200 \mathrm{mg}$ b.i.d. for 21-28 days per patient request. 


\section{Case Reports in Neurology}

\begin{tabular}{|c|c|}
\hline \multicolumn{2}{|c|}{ Case Rep Neurol 2016;8:151-155 } \\
\hline $10.1159 / 000447120$ & $\begin{array}{l}\text { (C) } 2016 \text { The Author(s). Published by S. Karger AG, Basel } \\
\text { www.karger.com/crn }\end{array}$ \\
\hline
\end{tabular}

Three weeks after discharge, the patient's follow-up visit had noted a complete resolution of his headache and facial pain. MRI with and without contrast revealed improvement in leptomeningeal enhancement. He continued to experience vision loss in his left eye with a visual acuity of 20/350. At an additional follow-up 2 months after discharge, continuous vitreous hemorrhage in his left eye was apparent on dilated funduscopic examination. Ophthalmology performed a pars plana vitrectomy, which helped clear infiltrations in the vitreous humor. Months after diagnosis and treatment, Candida albicans grew from vitreal cultures obtained from vitrectomy.

\section{Discussion}

Infectious agents are believed to enter the central nervous system hematogenously via the choroid plexus, which subsequently triggers an inflammatory cascade allowing for a disruption of the blood-brain barrier. Patients with suspected chronic infectious meningitis should receive an extensive workup including lumbar puncture, neuroimaging with MRI, and serum studies as indicated. Fungal infections reveal variable CSF findings, some of which may mimic tuberculosis [2,3]. Complications of meningitis include venous thrombosis, ventriculitis, cerebritis, vasculitis, and subdural/epidural empyema, all of which may be detected by MRI [2].

Candidal endophthalmitis is commonly associated with intravenous drug abuse, specifically brown heroin. The source of the infection is thought to be due to the manipulation of contaminated material used to dissolve the heroin. Patients most commonly present with gradual vision loss with or without ocular pain [4,5]. Although there is no established treatment protocol for candidal endogenous endophthalmitis, voriconazole is a newgeneration triazole antifungal that has been shown to achieve therapeutic intraocular levels with oral administration, and systemic antifungal therapy alone is usually sufficient in the absence of vitritis [6]. In the presence of vitreal lesions or vitritis, intravitreal injection of antifungal agents is usually added to systemic antifungal treatment, and vitrectomy can be performed for persistent vitreal opacities, vitritis or nonclearing vitreous hemorrhage [7].

\section{Statement of Ethics}

The authors have no ethical conflicts to disclose.

\section{Disclosure Statement}

There are no conflicts of interest to disclose.

\section{References}

1 Strang J, Keaney F, Butterworth G, Noble A, Best D: Different forms of heroin and their relationship to cook-up techniques: data on, and explanation of, use of lemon juice and other acids. Subst Use Misuse 2001;36:573-588.

-2 Hazany S, Go JL, Law M: Magnetic resonance imaging of infectious meningitis and ventriculitis in adults. Top Magn Reson Imaging 2014;23:315-325. 


\section{Case Reports in Neurology}

\begin{tabular}{l|l}
\hline $10.1159 / 000447120$ & $\odot 2016$ The Author(s). Published by S. Karger AG, Basel
\end{tabular}

www.karger.com/crn

Elfiky and Baldwin: Brown Heroin-Associated Candida albicans Ventriculitis and Endophthalmitis Treated with Voriconazole

-3 Voice RA, Bradley SF, Sangeorzan JA, Kauffman CA: Chronic candidal meningitis: an uncommon manifestation of candidiasis. Clin Infect Dis 1994;19:60-66.

4 Patel SN, Rescigno RJ, Zarbin MA, Langer P, Bhagat N: Endogenous endophthalmitis associated with intravenous drug abuse. Retina 2014;34:1460-1465.

5 Bisbe J, et al: Disseminated candidiasis in addicts who use brown heroin: report of 83 cases and review. Clin Infect Dis 1992;15:910-923.

-6 Motukupally SR, et al: Ocular infections caused by Candida species: type of species, in vitro susceptibility and treatment outcome. Indian J Med Microbiol 2015;33:538-546.

7 Martinez-Vazquez C, Fernandez-Ulloa J, Bordon J, Sopena B, de la Fuente J, Ocampo A, et al: Candida albicans endophthalmitis in brown heroin addicts: response to early vitrectomy preceded and followed by antifungal therapy. Clin Infect Dis 1998;27:1130-1133.

$\$ 8$ Baldwin K, Whiting C: Chronic meningitis: simplifying a diagnostic challenge. Curr Neurol Neurosci Rep 2016;16:30.

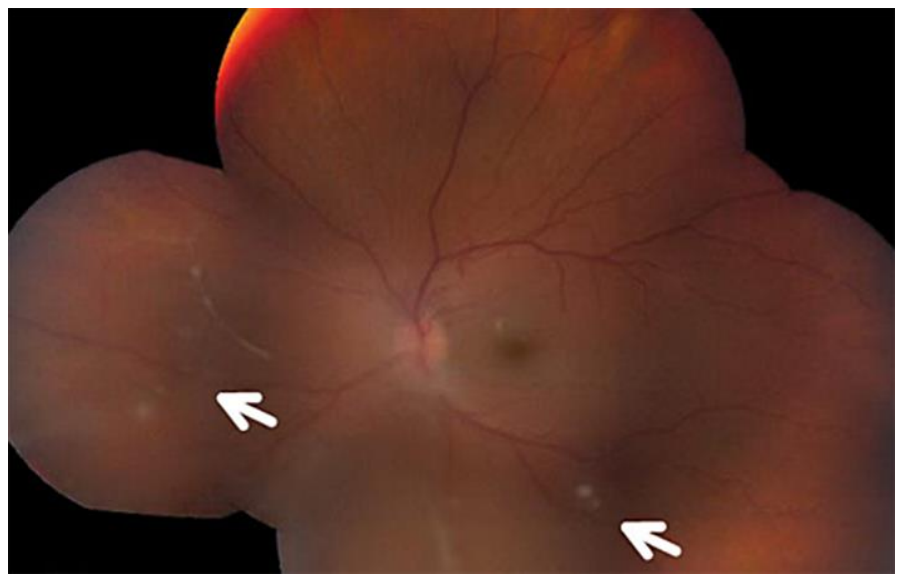

Fig. 1. Montage of left eye shows discrete fungal lesions in the vitreous with surrounding vitritis, optic nerve edema, and some folds in the macula. 


\section{Case Reports in Neurology}

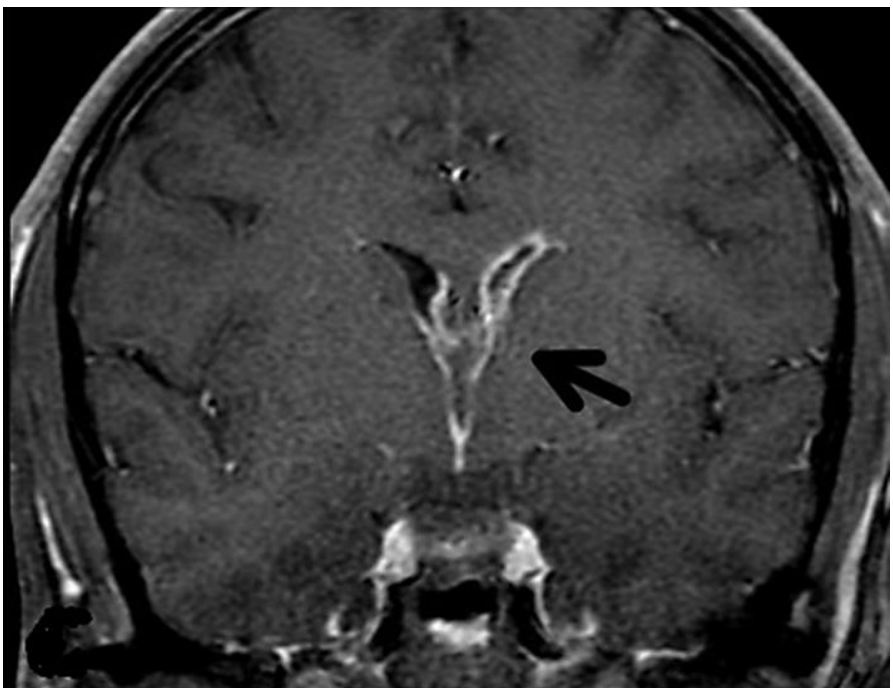

Fig. 2. MRI with and without gadolinium-based contrast depicts abnormal signal and pathological enhancement outlining all ventricles, especially the left lateral ventricle. These images were borrowed from another publication [8]; however, the images are taken from this reported patient case. 